\title{
Direct atomistic defect observations by depth sectioning and dynamic STEM
}

Ryo Ishikawa ${ }^{1}$, Naoya Shibata ${ }^{2}$ and Yuichi Ikuhara ${ }^{1}$

${ }^{1}$ Institute of Engineering Innovation, University of Tokyo, Tokyo, Japan, ${ }^{2}$ Institute of Engineering Innovation, University of Tokyo, United States

Since the invention of multi-poles-based aberration correctors, the ability of electron microscopy is significantly improved and the observations with sub-angstrom spatial resolution is routinely available. The spatial resolution in scanning transmission electron microscopy (STEM) is now reached into $40.5 \mathrm{pm}$ - less than half an angstrom - [1], which might be sufficient to directly determine the local atomic and electronic structures of materials. However, sub-angstrom resolution is only valid in the projected two dimensions, and the depth resolution along the axial direction is still far from atomic resolution. To determine atomistic defect structures in full three dimensions, it is therefore necessary to improve the depth resolution in STEM. The other critical issue in STEM is temporal resolution, and the most of atomicscale in-situ observations have been performed by TEM rather than STEM. This is because the temporal resolution in STEM is still in the order of seconds. To extend the capability of atomic-resolution STEM imaging to dynamic observations, it is prerequisite to develop a new faster scanning probe system. In this study, we will discuss our recent progress in (i) depth sectioning with large-angle illumination STEM [2] and (ii) high speed STEM imaging [3].

According to the principle in optics, the depth resolution in STEM can be improved as a function of illumination angle $\left(\lambda / \alpha^{2}\right)$. We have therefore installed a Delta-type corrector into JEM-ARM300CF [1], which significantly increase the flat region of Ronchigram up to $70 \mathrm{mrad}$. However, with larger illumination angles, it requires to rigorously remove residual geometric aberrations. The measurement accuracy of the lower orders of aberrations is not so high in conventional Ronchigram algorithms, we have therefore developed a method to minimize residual aberrations by using atomic-resolution STEM images [4]. By implementing the automated aberration correction system, it becomes possible to acquire atomicresolution STEM images with the illumination angle of $63 \mathrm{mrad}$ (Fig. 1). We then achieve the depth resolution of $2.1 \mathrm{~nm}$ with $\alpha$ of $63 \mathrm{mrad}$ [2].

In a current fast STEM image acquisition, an acquisition and fly-back times are typically $1 \mu$ s/pix and 500 $\mu \mathrm{s}$, respectively and the acquisition time per frame is $518 \mathrm{~ms}$ with the image size of $512 \times 512$ pixels. To significantly improve the temporal resolution, we have developed a new scanning probe system, where the total inductance of the scanning coils are 240 times smaller than in previous coils. The acquisition and fly-back times are $83 \mathrm{~ns} /$ pix and $35 \mu \mathrm{s}$, leading to 25 frame per second with the image size of $512 \times 512$ pixels. We will discuss the capability of high spatiotemporal-resolution STEM imaging, as an example of Pt nanoparticle atomic-scale motion on $\mathrm{TiO}_{2}$ (110) surface (Figure 2). 


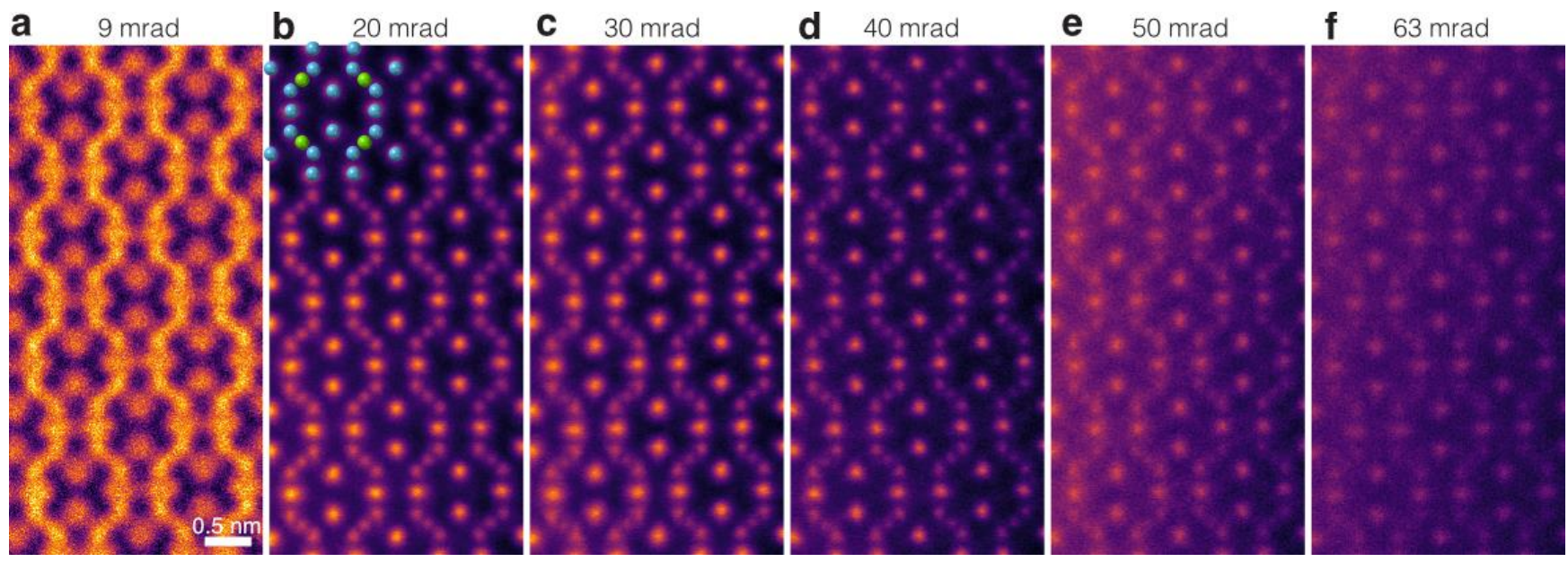

Figure 1. Atomic-resolution ADF STEM images of CoSb3 viewed along the [001] direction, where the illumination semi-angles are (a) $9 \mathrm{mrad}$, (b) $20 \mathrm{mrad}$, (c) $30 \mathrm{mrad}$, (d) $40 \mathrm{mrad}$, (e) $50 \mathrm{mrad}$ and (f) 63 mrad, respectively.
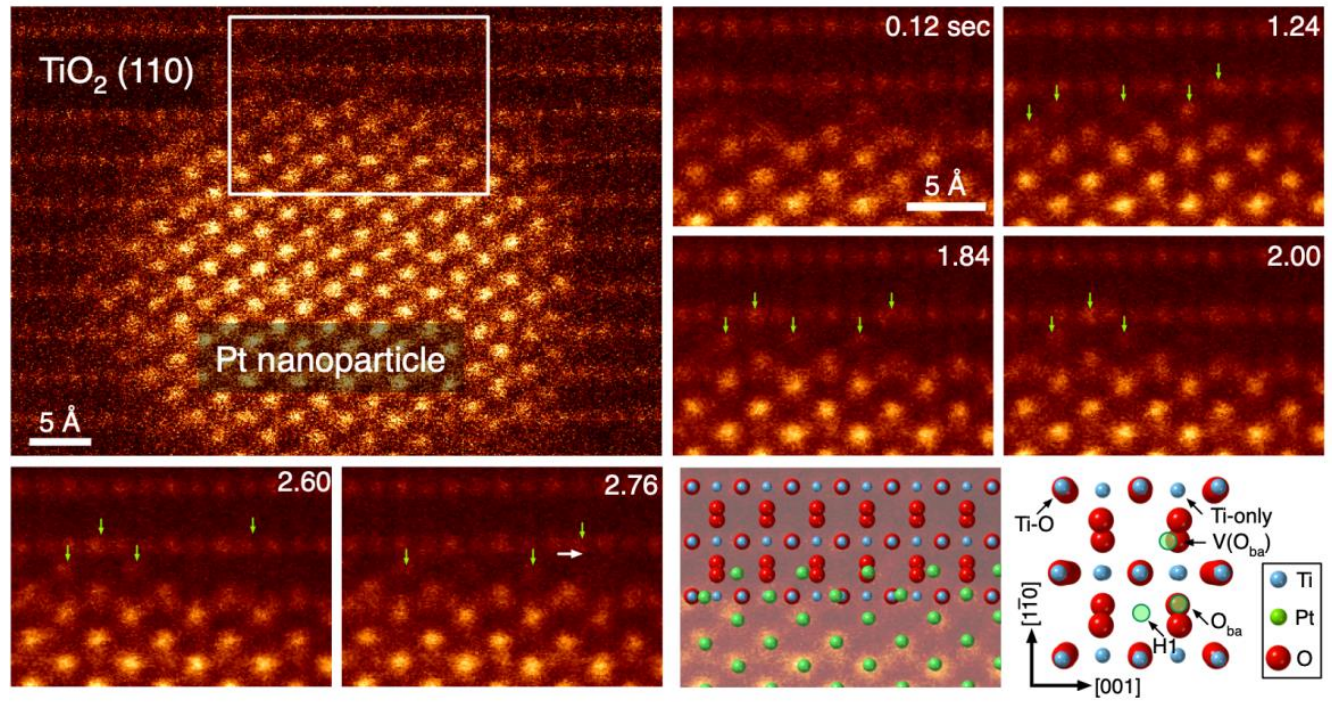

Figure 2. Time-sequential ADF STEM images of Pt-nanoparticle on TiO2 (110) surface. Time stamps are given in the top-right of respective frame. The green arrows indicate the locations of Pt single atoms. The structure model is given in the bottom right.

References

[1] S. Morishita et al, Microscopy 6746 (2018).

[2] R. Ishikawa et al, Phys. Rev. Appl. 13034064 (2020).

[3] R. Ishikawa et al, Microscopy 69240 (2020).

[4] R. Ishikawa et al, Ultramicroscopy 222133215 (2021).

[5] This work was partly supported by NEDO-RISING2 project (JPNP16001). 